\title{
Article \\ Role of Epstein-Barr Virus C Promoter Deletion in Diffuse Large B Cell Lymphoma
}

\author{
Seiyo Mabuchi ${ }^{1,2,+}{ }^{\dagger}$ Fumiya Hijioka ${ }^{1,+}{ }^{\dagger}$, Takahiro Watanabe ${ }^{1} \mathbb{1}$, Yusuke Yanagi ${ }^{1}$, Yusuke Okuno ${ }^{3}$, \\ H. M. Abdullah Al Masud ${ }^{1,4}(\mathbb{D})$, Yoshitaka Sato ${ }^{1}\left(\mathbb{D}\right.$, Takayuki Murata ${ }^{1,5, *}$ and Hiroshi Kimura ${ }^{1, *}$ \\ 1 Department of Virology, Nagoya University Graduate School of Medicine, Nagoya 466-8550, Japan; \\ mabuchi.seiyo@med.nagoya-u.ac.jp (S.M.); fhijioka@mail.biken.or.jp (F.H.); \\ t.nabe.watanabe@med.nagoya-u.ac.jp (T.W.); yusuke.yanagi@med.nagoya-u.ac.jp (Y.Y.); \\ masud.mbio@cu.ac.bd (H.M.A.A.M.); yssato@med.nagoya-u.ac.jp (Y.S.) \\ 2 Department of Pathology and Laboratory Medicine, Nagoya University Graduate School of Medicine, \\ Nagoya 466-8550, Japan \\ 3 Medical Genomics Center, Nagoya University Hospital, Nagoya 466-8550, Japan; \\ yusukeo@med.nagoya-u.ac.jp \\ 4 Department of Microbiology, Faculty of Biological Sciences, University of Chittagong, \\ Chattogram 4331, Bangladesh \\ 5 Department of Virology and Parasitology, Fujita Health University School of Medicine, \\ Toyoake 470-1192, Japan \\ * Correspondence: tmurata@fujita-hu.ac.jp (T.M.); hkimura@med.nagoya-u.ac.jp (H.K.) \\ + Equal contribution.
}

check for updates

Citation: Mabuchi, S.; Hijioka, F.; Watanabe, T.; Yanagi, Y.; Okuno, Y.; Masud, H.M.A.A.; Sato, Y.; Murata, T.; Kimura, H. Role of Epstein-Barr Virus C Promoter Deletion in Diffuse Large B Cell Lymphoma. Cancers 2021, 13, 561. https://doi.org/ 10.3390/cancers13030561

Academic Editor: Marc Blondel

Received: 11 November 2020

Accepted: 27 January 2021

Published: 1 February 2021

Publisher's Note: MDPI stays neutral with regard to jurisdictional claims in published maps and institutional affiliations.

Copyright: (c) 2021 by the authors. Licensee MDPI, Basel, Switzerland. This article is an open access article distributed under the terms and conditions of the Creative Commons Attribution (CC BY) license (https:// creativecommons.org/licenses/by/ $4.0 /)$.
Simple Summary: The C promoter of Epstein-Barr virus is assumed to be important for B cell growth and transformation. However, we present evidence that promoter activity is not only unneeded for transformation but also that absence of the promoter increased the transformation activity of the virus. We found that the $\mathrm{C}$ promoter was lost in some Epstein-Barr virus-associated lymphoma specimens. Therefore, deletion of the promoter could partially account for the tumorigenesis of Epstein-Barr virus-associated lymphomas.

Abstract: The Epstein-Barr virus (EBV) is the cause of several malignancies, including diffuse large B cell lymphoma (DLBCL). We recently found that EBV genomes in EBV-positive cancer specimens have various deletions (Okuno et al. Nat Microbiol. 2019). Here, we focus on the deletion of $C$ promoter (Cp), which transcribes EBV nuclear antigen (EBNA) genes in type III latency. The Cp deletion found in a DLBCL patient (332 bp) was introduced into EBV-BAC of the B95-8 strain. Interestingly, the $\mathrm{dCp}$ virus transformed B cells more efficiently than WT and revertant strains. Deletion of Cp also promoted tumor formation and severe pathogenicity in a mouse xenograft model. RNA sequencing and qRT-PCR analyses revealed that Cp transcription was undetectable in the dCp cells. Instead, transcription from the $\mathrm{W}$ promoter $(\mathrm{Wp})$, an alternative promoter for EBNA, was activated in the $\mathrm{dCp}$ mutant. We also found that the expression of latent membrane protein 2A (LMP2A) was somehow induced in the dCp mutant. Double knockout of Cp and LMP2A indicated that LMP2A is crucial for $\mathrm{B}$ cell transformation, but the increased transformation induced by $\mathrm{Cp}$ deletion cannot be explained by LMP2A alone. We also tested the effect of an anti-apoptotic viral BCL2 homolog, BHRF1, because its expression was reportedly induced more efficiently by that of Wp. However, increased growth transformation via $\mathrm{Cp}$ deletion was not due to the BHRF1 gene. Taken together, the results indicated that deletion of a specific region in $\mathrm{Cp}$ increased in vitro transformation and the rate of progression of EBV-positive lymphoproliferative disorders in vivo. Our data suggest that genomic alteration not only of the host but also the virus promotes EBV-positive tumor generation and expansion, although the molecular mechanism underlying this phenomenon is still unclear. However, LMP2A and BHRF1 are not involved.

Keywords: EBV; DLBCL; C promoter; LMP2A; BHRF1; growth transformation 


\section{Introduction}

The Epstein-Barr virus (EBV) is a member of the human gamma-herpesvirus family, which was first discovered in endemic Burkitt lymphoma (BL) cells. EBV contains a large double-stranded DNA genome ( 170 kbps) encoding over 80 genes and 40 mature microRNAs (miRNAs) and noncoding RNAs. It is a ubiquitous virus present in over $90 \%$ of the worldwide population and primarily infects B cells via the saliva during childhood. Most cases are asymptomatic, but it occasionally manifests as infectious mononucleosis.

After the primary infection, the virus suppresses the expression of most viral genes, hiding predominantly in memory B cells without virus production nor presenting obvious symptoms (latent infection). Latent EBV expresses its genes in different patterns according to latency programs, categorized as type 0-III. For example, EBV can infect peripheral blood B cells in vitro, resulting in transformation into continuously proliferating lymphoblastoid cell lines (LCLs). The LCLs take type III latency and express all latent EBV genes. Regardless, after some time, EBV can reactivate from latency and enter its lytic cycle, resulting in the production of progeny virus.

A small but a significant number of EBV-positive people develop lymphomas, including BL, Hodgkin lymphoma, diffuse large B cell lymphoma (DLBCL), chronic active EBV infection (CAEBV), extranodal natural killer (NK)/T cell lymphoma, nasal type (ENKL), nasopharyngeal carcinoma, and gastric cancer. The reason why these cancers affect some EBV-positive patients is still incompletely understood [1,2].

EBV is associated with tumor growth and proliferation through multiple mechanisms. EBV contains oncogenes such as latent membrane protein 1 (LMP1) and LMP2A. These genes mimic CD40 and BCR, respectively, and activate downstream signaling pathways [3,4]. LMP1 and LMP2A have been shown to mediate Reed-Sternberg cell survival and proliferation in Hodgkin lymphoma. Another EBV gene, BNRF1, induces genomic alterations and chromosomal instability in the host genome through amplifying centrosome activity, resulting in genomic mutations [5]. EBV nuclear antigen 2 (EBNA2) binds to super-enhancer regions and modifies gene transcription, such as by upregulating the MYC oncogene [6]. LMP1 and EBNA2 allow the virus to escape immune detection through upregulation of PD-L1 [7,8].

Until recently, it was believed that EBV in cancers always had a complete set of genes in the full-length viral genome without any deletions, except a few rare cases, such as Wp-restricted BL [9]. However, we recently found that the viral genome in EBV-associated lymphomas and lymphoproliferative disorders bears several types of deletion $[10,11]$. Especially, we found deletion of the EBV C promoter (Cp) in cases of DLBCL, ENKL and CAEBV (Figure 1). Cp is an EBV promoter that mediates the transcription of EBNAs in type III latency cells, such as LCLs. Upon EBV infection of B cells, the W promoter $(\mathrm{Wp})$ is activated and produces EBNA proteins, including EBNA2. As the expression of EBNA2 protein increases, it activates $\mathrm{Cp}$ and other downstream promoters and suppresses $W p[12,13]$. Hence, the $W p$ is activated for expression of EBNAs immediately after infection, but then within a week or two, the Cp takes over the expression of EBNAs, although the physiological significance of this promoter switching is not understood yet.

We are interested in the role of $\mathrm{Cp}$ deletion in the generation of EBV-associated tumors. To elucidate the mechanism, we introduced a Cp deletion into an EBV-bacterial artificial chromosome (BAC) and compared the phenotype between $\mathrm{Cp}$-deficient $(\mathrm{dCp})$ virus and the wild-type (WT) virus. Interestingly, disruption of $\mathrm{Cp}$ resulted in a 10-fold higher rate of B cell growth transformation. We examined the role of LMP2A and BHRF1 in the increased immortalization seen in the $\mathrm{dCp}$ virus infection but found no connection. Therefore, deletion of the Cp in a DLBCL was confirmed to be beneficial for tumorigenesis, but the molecular mechanism remains unknown. 

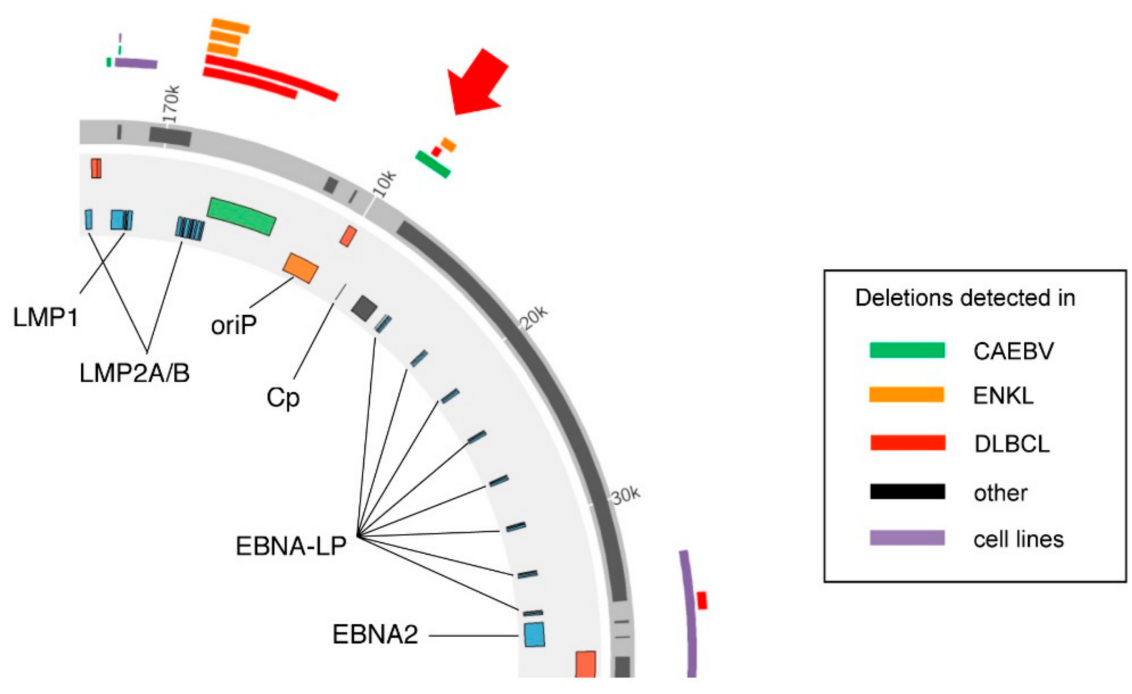

Figure 1. Deletion of the Epstein-Barr virus (EBV) genome in EBV-positive cancer specimens. Map of the deletions in $\mathrm{C}$ promoter $(\mathrm{Cp})$ found in our previous study (Okuno et al. Nature Microbiology, 2019). These deletions were present in chronic active EBV infection (CAEBV), EBV-positive diffuse large B-cell lymphoma (DLBCL) and extranodal natural killer (NK)/T-cell lymphoma, nasal type (ENKL) patients. Modified from (Murata et al. Reviews in Medical Virology, 2020).

\section{Results}

\subsection{Construction of Cp-Deficient EBV-BAC Strain}

Previously, we demonstrated that EBVs latently infected in a portion of human lymphoma specimens have intragenic deletions [10]. These deletions were most frequently found in the BamHI-A rightward transcript (BART) region and essential lytic genes. Loss of the BART region increased lytic gene expression and tumor formation in a mouse xenograft model [14]. Disruption of BALF5 showed a similar phenotype [10]. Several of the deletions found in CAEBV, ENKL, and DLBCL tissue biopsies affected Cp (Figure 1). The discovery of these deletions in clinical specimens was surprising because it seemed to contradict previous reports that the loss of $\mathrm{Cp}$ expression resulted in lower B cell growth transformation activity $[15,16]$. As CAEBV and ENKL affect both T and NK cells, we decided to focus on the $\mathrm{Cp}$ deletion seen in the DLBCL specimen.

To investigate the effect of $\mathrm{Cp}$ deletion on the EBV life cycle, we constructed a Cpdeficient recombinant EBV using the EBV-BAC system (Figure 2). We first inserted the neomycin-resistant/streptomycin-sensitive (Neo/St) gene cassette into WT EBV-BAC DNA (B95-8 strain) using homologous recombination. Then the Neo/St cassette was replaced with a $\mathrm{Cp}$ sequence including a deletion, i.e., dCp. A revertant (rev) strain was prepared by inserting and then removing the Neo/St cassette (Figure 2A). To confirm the integrity of the viral genome, recombinant EBV-BAC DNA was digested with BamHI or EcoRI, and the digested products were examined by electrophoresis (Figure 2B,C). The band patterns of the three strains (WT, $\mathrm{dCp}$, and rev) were identical aside from a digested product near $9.4 \mathrm{kbp}$ that was shortened in the $\mathrm{dCp}$ by digestion with BamHI (arrowhead), reflecting that the intended $\mathrm{Cp}$ deletion was achieved. These results indicate that the recombinant viral genomes were intact and did not have obvious unexpected deletions or insertions.

These EBV-BAC DNAs were transfected to HEK293 cells by lipofection. Hygromycin resistant green fluorescent protein (GFP)-positive colonies were collected, in which the recombinant EBV genome is latently maintained. Cells were transfected with a BZLF1expressing vector to activate the lytic cycle of EBV and induce progeny virus production. We confirmed that deletion of the $\mathrm{Cp}$ had little or no effect on the production of progeny EBV production, at least in HEK293 cells (not shown). The viral titers were determined by infecting $\mathrm{Akata}(-)$ cells and used for the transformation assays. 

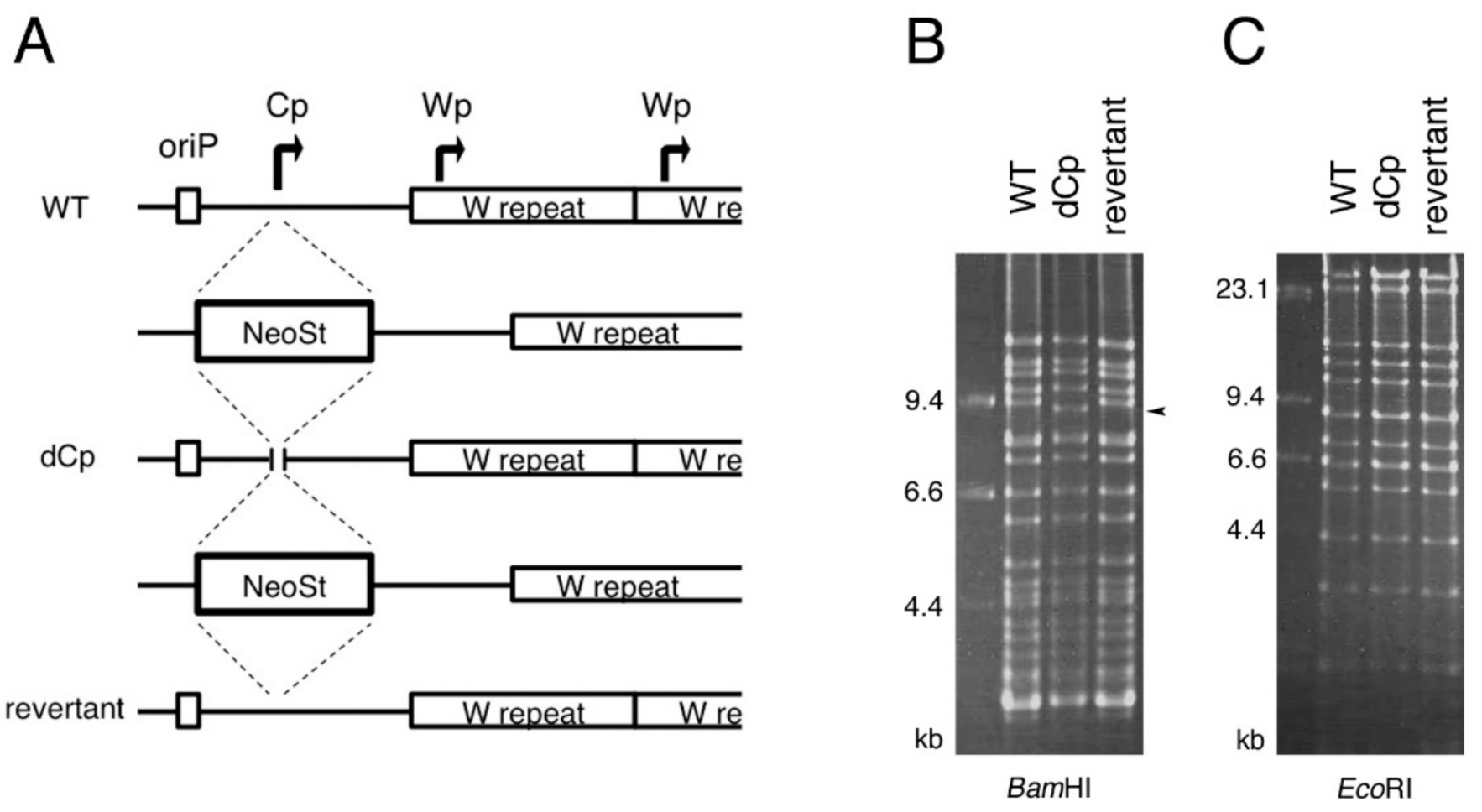

Figure 2. Construction of the $\mathrm{C}$ promoter deletion $(\mathrm{dCp})$ and revertant (rev) EBV mutant constructs using the EBV-bacterial artificial chromosome (BAC) system. (A) Schematic diagram of the EBV-BAC recombination. The Neo/St cassette was inserted first in the $\mathrm{Cp}$. Neo/St cassette was replaced with a Cp sequence with deletion from 11,145 to 11,476 (332 bp) of KC207813.1. The Neo/St cassette was reinserted again and then replaced with the wild-type (WT) Cp sequence to construct the rev virus. $(\mathrm{B}, \mathrm{C})$ Recombinant EBV-BAC DNA was digested by BamHI and EcoRI, and the products were examined by agarose gel electrophoresis. Note that the digested product near $9.4 \mathrm{kbp}$ was shortened in the $\mathrm{dCp}$ virus (indicated by arrowhead), reflecting the deletion.

\subsection{Cp Deletion Increases B Cell Transformation Efficiency}

To examine the role of $\mathrm{Cp}$ deletion in $\mathrm{B}$ cell growth transformation, we infected peripheral blood mononuclear cells (PBMCs) from healthy donors with the three viral strains (WT, $\mathrm{dCp}$ and rev) described above (Figure 3). At a multiplicity of infection (MOI) of 0.2, WT and rev strains caused obvious cell clump formation in about $50 \%$ of wells, compared to $100 \%$ of the wells treated with the $\mathrm{dCp}$ virus at day 10 (Figure $3 \mathrm{~A}$ ). A similar trend was observed in the samples infected at a lower MOI (0.02) (Figure 3B). Transformation efficiency was calculated after 3 weeks of incubation. The log transformation units per milliliter were 2.38, 3.25 and 2.25 in WT, dCp, and rev, respectively (Figure 3C). Reproducibility was confirmed by using PBMCs from a second donor, where the WT, dCp, and rev EBVs exhibited 1.43, 2.43 and $1.55 \log$ transformation units per milliliter, respectively (Figure 3D). Therefore, the dCp EBV caused increased B cell transformation compared to controls. 


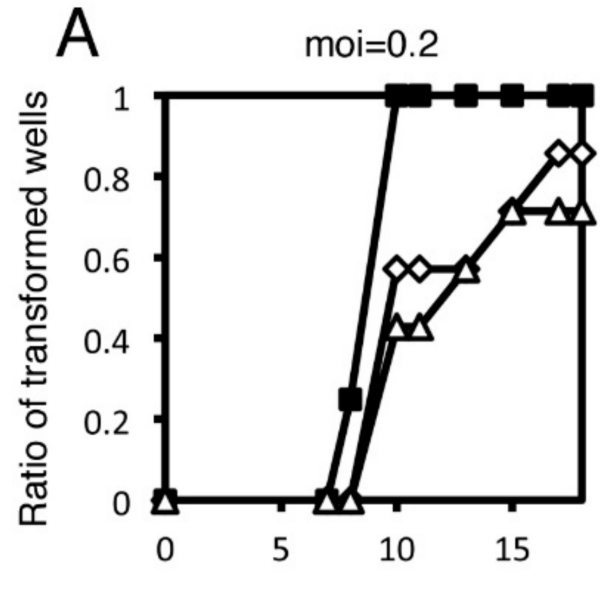

Days after infection

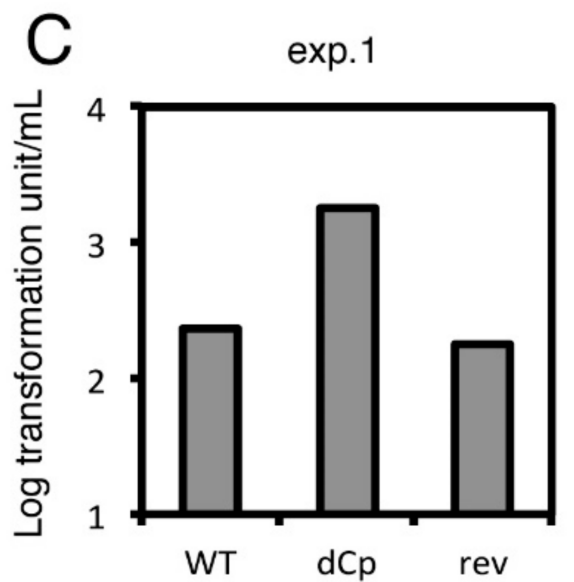

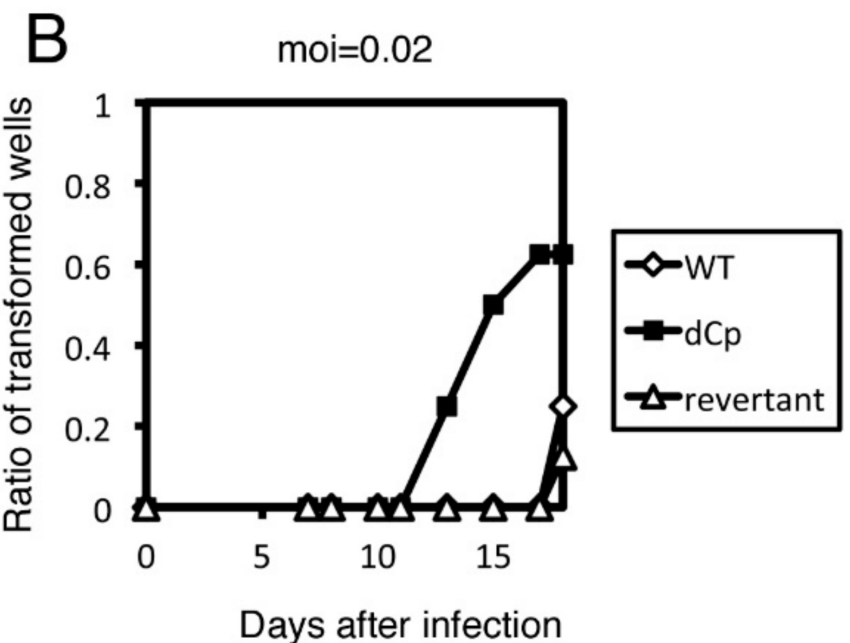

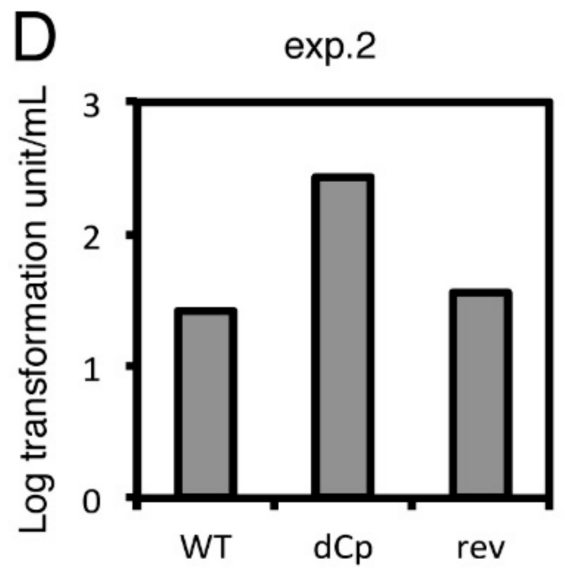

Figure 3. Transformation of WT, dCp and rev viruses. Recombinant viruses (WT, dCp and rev) were produced from HEK293 cells. After titer normalization of the viruses, 10-fold serial dilutions were prepared and used to separately infect peripheral blood mononuclear cells (PBMCs). For each dilution, 8 wells were prepared. (A,B) Ratio of transformed wells with a multiplicity of infection (MOI) of 0.2 (A) and 0.02 (B) over time. (C,D) Transformation efficiency at 3 weeks post-infection. Two independent experiments were carried out using PBMCs from two healthy donors (exp. 1 and exp. 2).

\section{3. $d C p$ Virus Increased Pathogenicity in an EBV-Associated LPD Mouse Model}

We next examined the influence of $C p$ deletion in vivo using a mouse xenograft model. Human cord blood mononuclear cells were infected with WT or dCp virus and immediately injected intraperitoneally into NOG mice. Mice receiving $\mathrm{dCp}$ virus-infected cells showed a higher mortality rate compared to controls (Figure 4A) and more severe weight loss (Figure 4B), although the body weight loss was not statistically significant. Tumors formed in the pancreas of the mice were then subjected to hematoxylin-eosin (H-E) staining. Infection of either WT or dCp resulted in tumor formation, but the mice with the $d C p$ virus exhibited more extensive infiltration of tumor cells (Figure $4 C, D$ ). These results imply that $\mathrm{Cp}$ deletion also plays an important role in EBV-associated tumor progression in vivo. We here analyzed tumors in the pancreas because they were macroscopically detected easily than those in other organs, such as the spleen and liver, but there were tumors in other organs, too. 

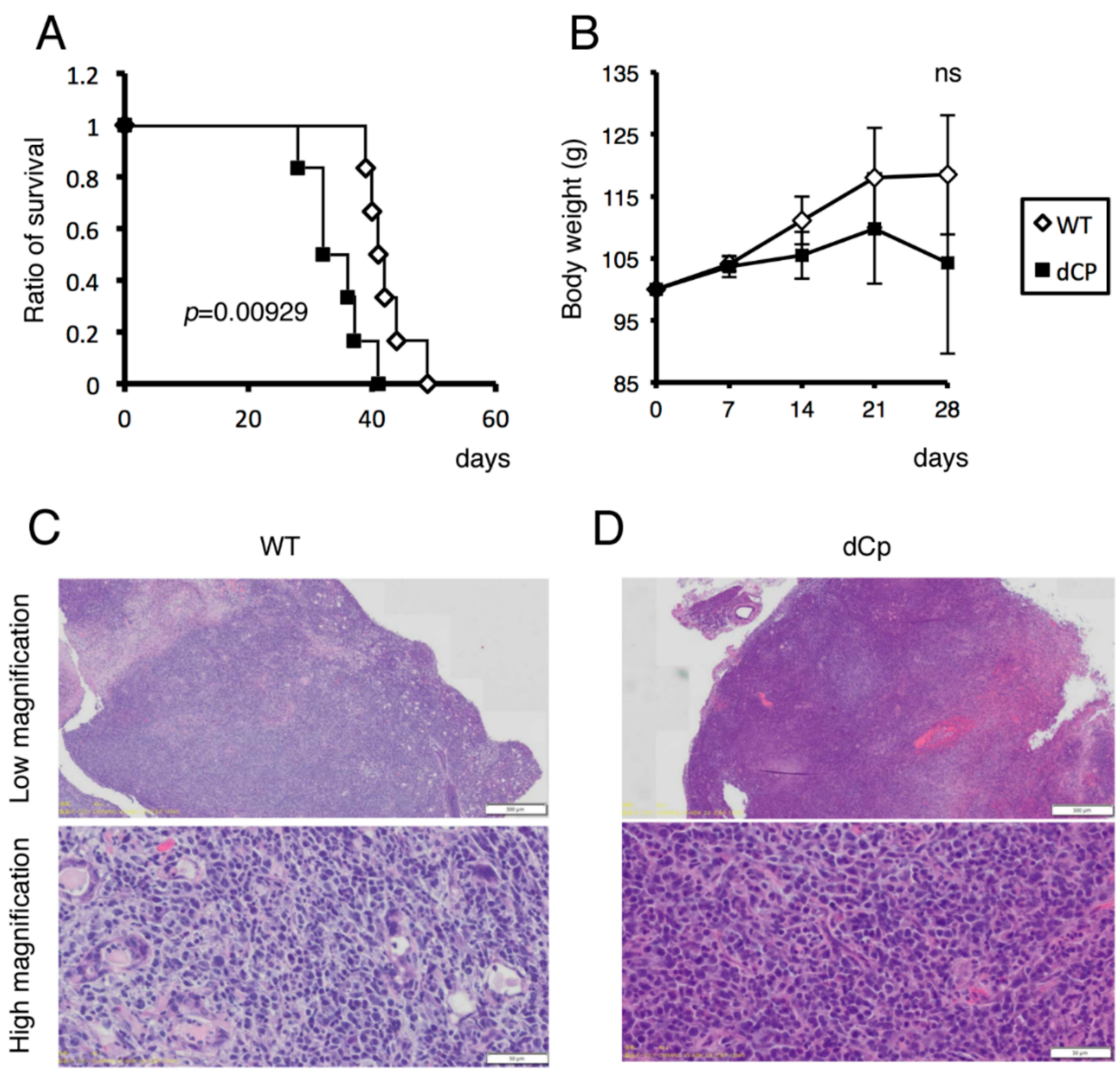

Figure 4. Survival of xenograft mice with EBV-associated lymphoproliferative disorder. First, $1.0 \times 10^{7}$ human cord mononuclear blood cells were each infected with $1.0 \times 10^{4}$ viruses $(\mathrm{WT}, \mathrm{dCp})$ and immediately transplanted intraperitoneally into NOG mice $(n=6)$. (A,B) Survival ratio (A) and body weight (B) of the mice were plotted. (C,D) Microscopic images of H-E staining of tumors in the pancreas of the mice infected with WT (C) or dCp (D) virus.

\subsection{LCLs with the dCp EBV Show Increased Expression of LMP2A and LMP2B}

To gain insight into the mechanism underlying how $\mathrm{Cp}$ deletion enhanced $\mathrm{B}$ cell growth transformation, we next investigated the gene expression profile of LCLs infected with the WT, dCp and rev viruses using RNA sequencing (RNA-seq). The RNA-seq analysis clearly showed that LCLs infected with the $\mathrm{dCp}$ virus utilized the Wp instead of $\mathrm{Cp}$ for transcription of EBNAs (Figure 5A), as reported [16]. Figure 5B-H summarizes fragments per kilobase of exon per million reads mapped (FPKM) values of the LCLs, which reflect expression levels of mRNAs. Transcript of EBNA-LP was markedly induced in the $\mathrm{dCp}$ (Figure $5 B$ ), which is convincing because the $d C p$ virus utilizes the $W p$ instead of $C p$, and the exons of EBNA-LP are mapped to the BamHI-W region. In addition, EBNA-LP of B95-8 strain EBV is not functional due to a stop codon insertion [17], indicating that induction in EBNA-LP level does not account for the increased transformation by $\mathrm{Cp}$ deletion. Levels of the EBNA2 gene were comparable, possibly because the activity of the Wp in the $\mathrm{dCp}$ virus was high enough to recover the loss of the Cp. Expression of BHRF1 was slightly higher in the dCp LCLs (Figure 5D), while those of EBNA3A and LMP1 were lower due to unknown reasons (Figure 5D-F). Interestingly, we found that two LMP2 genes, namely the LMP2A, were notably induced (Figure 5G,H). 

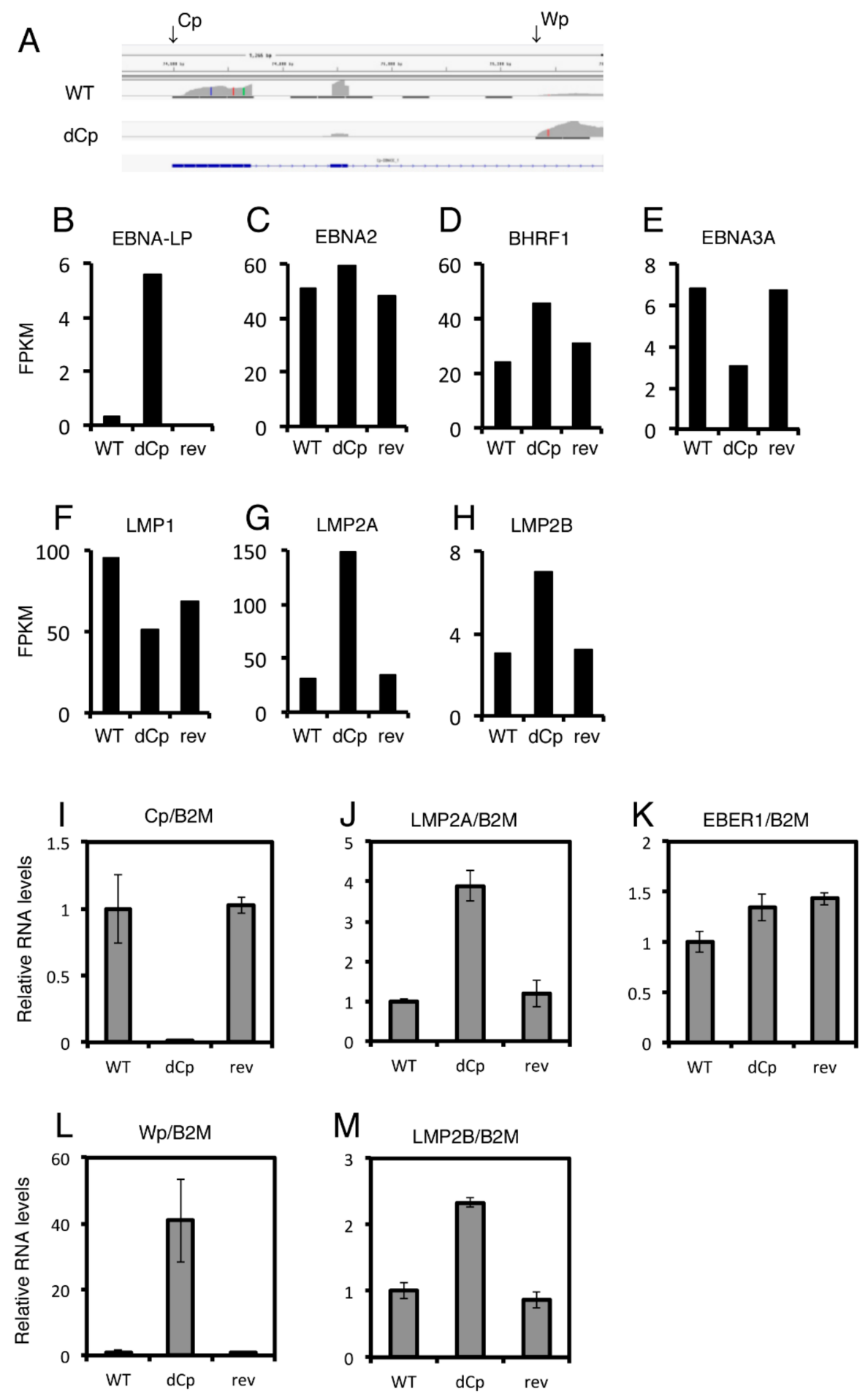

Figure 5. RNA sequencing (RNA-seq) and qRT-PCR of WT, $\mathrm{dCp}$ and rev virus-infected lymphoblastoid cell lines (LCLs). (A-H) RNA-seq analysis of infected LCLs. (A) read mapping of transcripts aligned to the $\mathrm{Cp} / \mathrm{Wp}$ region of EBV. (B-H) fragments per kilobase of exon per million reads mapped (FPKM) data of EBV genes are depicted. (I-M) qRT-PCR of the LCLs. Relative RNA levels were standardized by $\beta 2$-microglobulin (B2M). The mean and standard deviation values of three independent experiments are shown.

We then carried out a similar experiment, using primary B cells from a different donor, and analyzed the expression levels of EBV genes by qRT-PCR assays (Figure 5J-M). Figure 5I,L clearly showed that the Cp activity was lost, but instead, the Wp was induced in LCLs made by the $\mathrm{dCp}$ virus infection. Although the expression of the EBV noncoding RNA 
EBER1 was almost identical among the three viral strains (Figure 5K), dCp virus-infected LCLs showed higher LMP2A and LMP2B expression compared to controls (Figure 5J,M).

\subsection{LMP2A Does Not Account for the Enhanced Immortalization Efficiency in the $d C p$}

LMP2A and 2B are not necessary for B cell immortalization [18], but LMP2A is important for efficient immortalization [19]. LMP2A mimics the BCR signaling pathway and provides pro-survival and anti-differentiation signals to B cells [20]. We speculated that Cp deletion might increase LMP2A expression, resulting in increased B cell growth transformation efficiency. To test this possibility, using the EBV-BAC system, we deleted the first exon of the LMP2A gene in WT and $\mathrm{dCp}$ viral genomes to make an LMP2A-deficient virus (dLMP2A) and LMP2A/Cp-deficient virus (dCpdLMP2A) (Figure 6A). The digestion pattern of the recombinant EBV-BAC DNAs demonstrates that the DNAs were prepared as intended, without any deletions (Figure 6B).
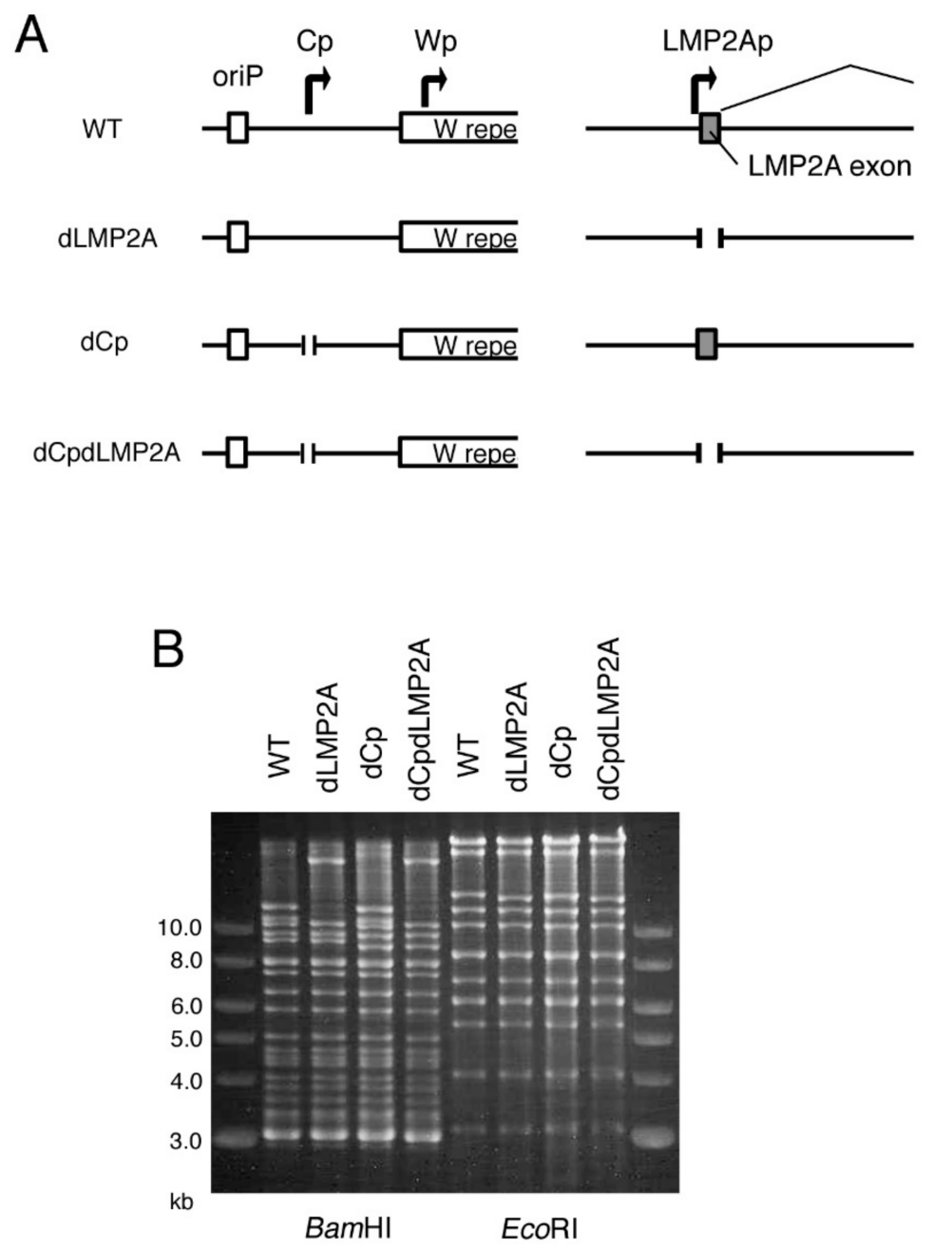

Figure 6. Construction of the latent membrane protein 2A-deficient (dLMP2A) and Cp/LMP2A double knockout (dCpdLMP2A) EBV mutants using the EBV-BAC system. (A) Schematic diagram of the EBV-BAC recombinant viruses. The first exons of the LMP2A gene of WT and dCp EBV constructs were removed to prepare dLMP2A and dCpdLMP2A, respectively. (B) Recombinant EBV-BAC DNA was digested by BamHI and EcoRI, and the products were examined by agarose gel electrophoresis.

After transfection into HEK293 cells, recombinant viruses were prepared and titrated, followed by normalization of the titer, and infected to PBMCs (Figure 7). Deletion of Cp in the background of the WT virus caused faster clump formation (Figure 7B), as we already showed above (Figure 3); Cp deletion in the LMP2A knockout virus background also 
promoted faster transformation. The transformation efficiency of the double knockout (dCpdLMP2A) was similar to that of WT and lower compared to the dCp mutant. Single knockout of LMP2A (dLMP2A) resulted in lower transformation efficiency than WT virus (Figure 7C,D). Since the promotion of growth transformation via $\mathrm{Cp}$ deletion took place even in the absence of LMP2A, LMP2A was not necessary for the promotion of growth transformation via $\mathrm{Cp}$ deletion. Therefore, we concluded that the mechanism of enhanced $\mathrm{B}$ cell activation by $\mathrm{Cp}$ deletion does not involve LMP2A.

A

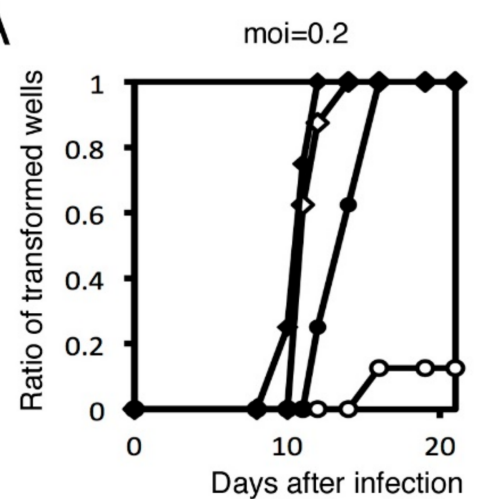

C

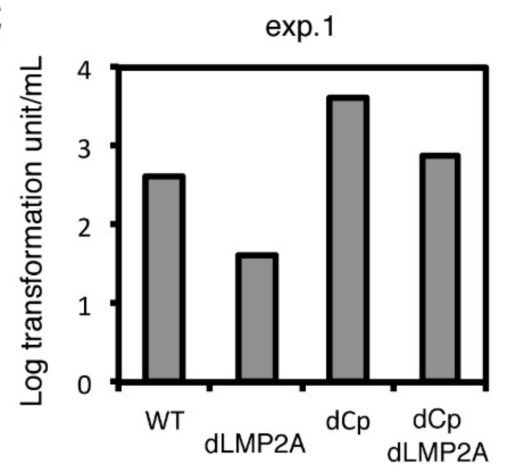

$\mathrm{B}$

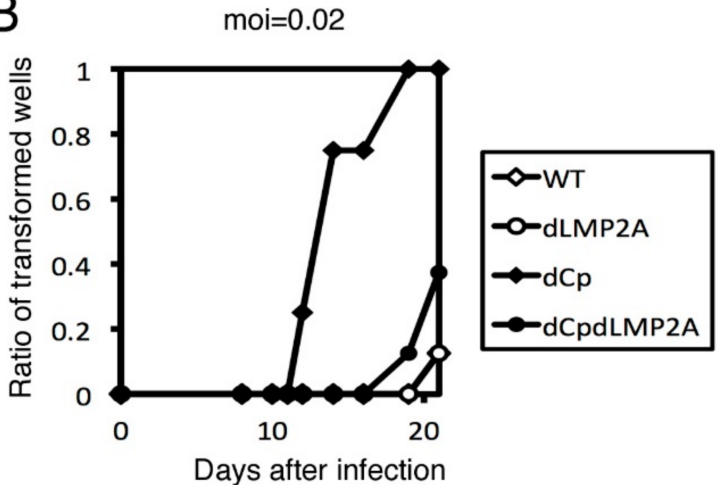

$\mathrm{D}$

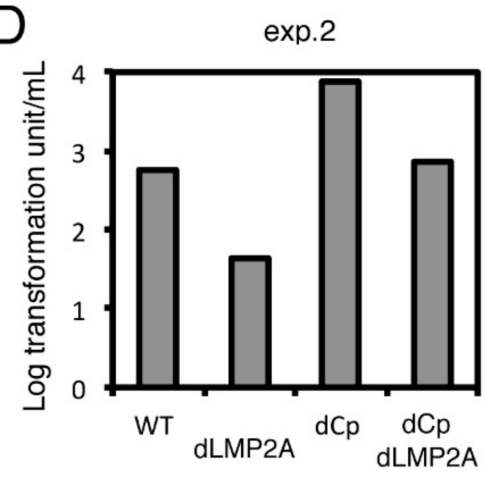

Figure 7. Transformation assay of dLMP2A and dCpdLMP2A viruses. Recombinant viruses (WT, dLMP2A, dCp, and dCpdLMP2A) were produced from HEK293 cells. After titer normalization of the viruses, 10-fold serial dilutions were prepared and used to separately infect PBMCs. For each dilution, 8 wells were prepared. (A,B) Ratio of transformed wells with a multiplicity of infection (MOI) of 0.2 (A) and 0.02 (B) over time. (C,D) Transformation efficiency at 3 weeks post-infection. Two independent experiments were carried out using PBMCs from two healthy donors (exp. 1 and exp. 2).

\subsection{Viral BCL2 Homolog BHRF1 Does Not Account for the Enhanced Transformation Efficiency} Seen in the $d C p$

BHRF1 is one of the EBV-coding BCL2 homologs. It functions as an anti-apoptotic protein by binding the pro-apoptotic proteins Bim, Puma and Bak [21-23]. BHRF1 is highly expressed by the Wp during primary infection [9] and enhances $B$ cell growth transformation efficiency [24]. Wp-restricted BL cell line P3HR-1 requires BHRF1 for its survival and proliferation [25]. Because the $\mathrm{dCp}$ virus activates the Wp instead of Cp (Figure 5), and, indeed, the BHRF1 level was marginally higher in the dCp LCLs (Figure 5D), we speculated that BHRF1 might play a role in the enhanced transformation efficiency of the $\mathrm{dCp}$ virus. To test this, using the EBV-BAC system, a stop codon was inserted into the BHRF1 exon in the WT and $\mathrm{dCp}$ viral genomes to generate the BHRF1-deficient virus (dBHRF1) and BHRF1/Cp-deficient virus (dCpdBHRF1) (Figure 8A). Recombinant EBV-BAC DNA was digested by BamHI or EcoRI, and the digested products were confirmed by electrophoresis (Figure 8B,C). 
A

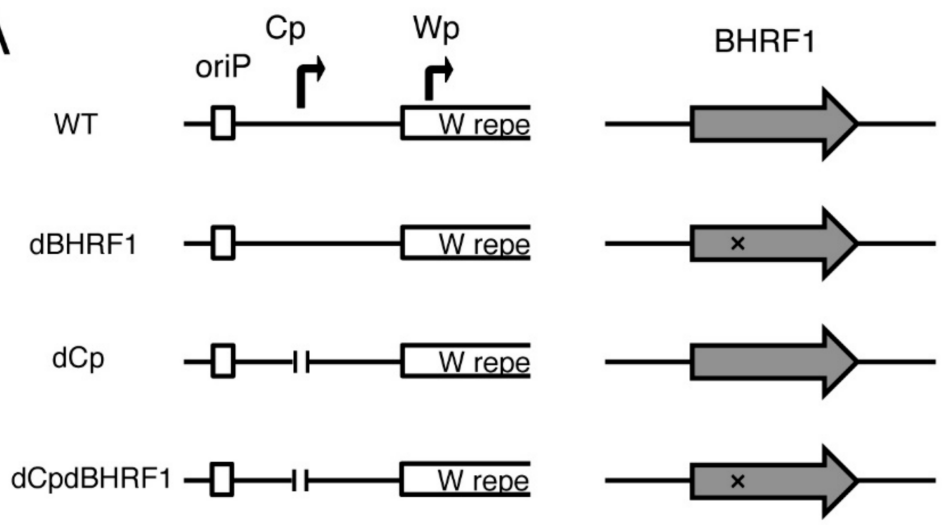

B
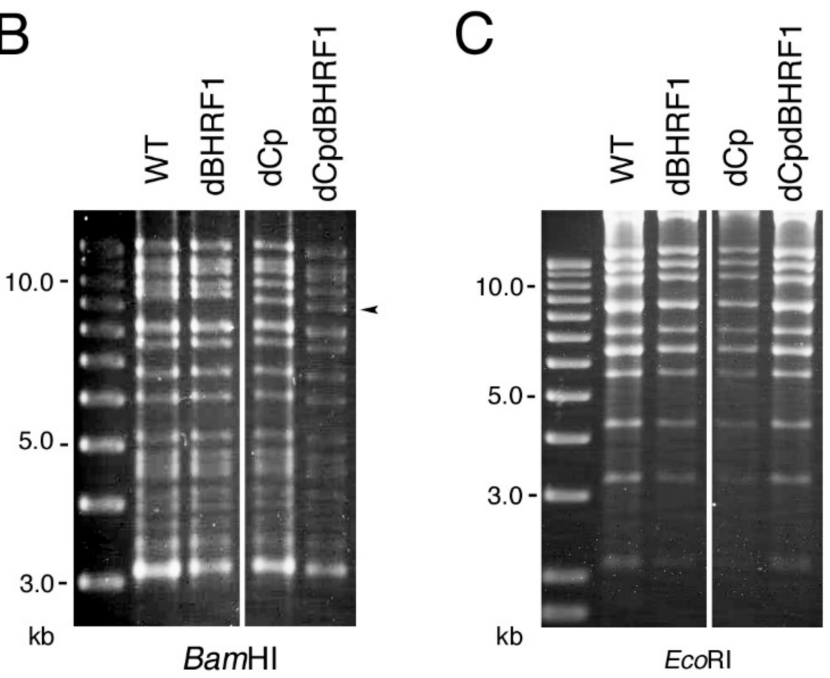

Figure 8. Construction of the BHRF1 deficient (dBHRF1) and Cp/BHRF1 double knockout (dCPdBHRF1) EBV mutants using the EBV-BAC system. (A) Schematic diagram of the EBV-BAC recombinant viruses. Two adenine residues were inserted between nucleotide 194 and 195 of BHRF1 amino acids to make a stop codon ( $\left.\mathrm{T}^{194} \mathrm{AA}\right)$. (B,C) Recombinant EBV-BAC DNA was digested by BamHI and EcoRI, and the products were examined by agarose gel electrophoresis.

We conducted the B cell growth transformation assay again (Figure 9) in a similar manner, except that we concentrated virus stock by ultracentrifuge before infection because transformation by the BHRF1 mutant was very low. Deletion of Cp increased transformation efficiency compared to WT, and BHRF1 knockout (dCpdBHRF1) lowered the transformation efficiency to some extent (Figure 9A-D). However, transformation in dBHRF1 virus was severely impaired compared to WT virus (Figure 9A-D). In exp. 2, clump formation was completely absent in the BHRF1 knockout samples (Figure 9D). Therefore, these results indicate that the viral BCL2 homolog, BHRF1, was also unable to explain the enhanced transformation efficiency in the $\mathrm{dCp}$ virus because the promotion of growth transformation by $\mathrm{Cp}$ deletion took place even in the absence of the BHRF1 gene (Figure 9). 
A

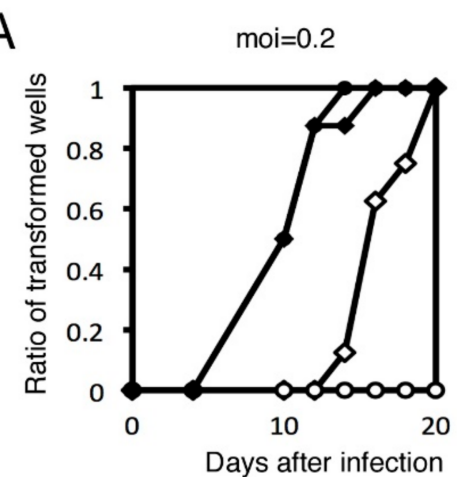

C

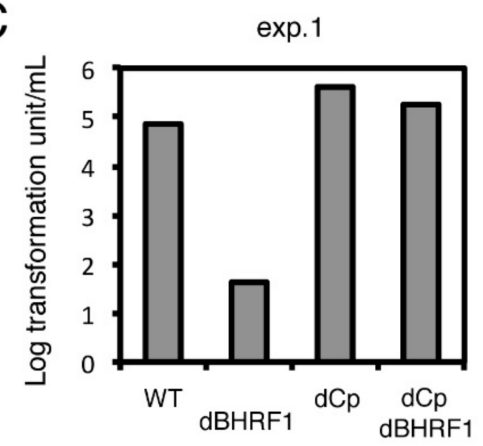

$\mathrm{B}$

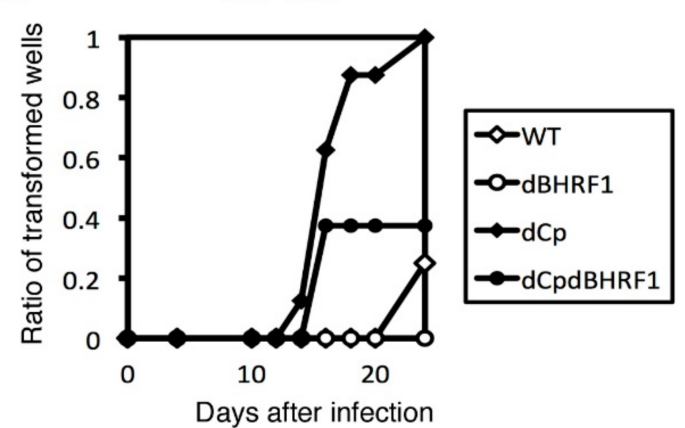

D

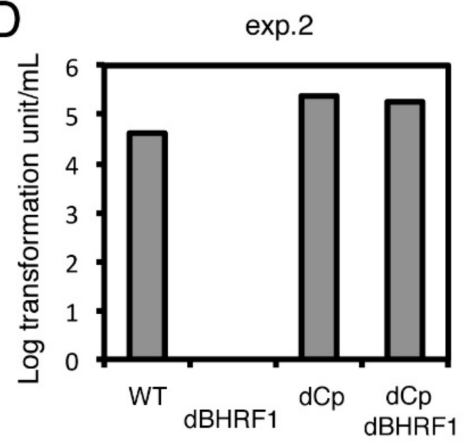

Figure 9. Transformation assay of dBHRF1 and dCpdBHRF1 viruses. Recombinant viruses (WT, dBHRF1, dCp, and dCpdBHRF1) were produced from HEK293 cells. After titer normalization of the viruses, 10-fold serial dilutions were prepared and used to separately infect PBMCs. For each dilution, 8 wells were prepared. (A,B) Ratio of transformed wells with a multiplicity of infection (MOI) of 0.2 (A) and 0.02 (B) over time. (C,D) Transformation efficiency at 3 weeks post-infection. Two independent experiments were carried out using PBMCs from two healthy donors (exp. 1 and exp. 2).

\section{Discussion}

Our results demonstrate that deletion of the EBV Cp enhances B cell growth transformation in vitro and results in faster expansion of EBV-associated lymphoproliferative disorders in vivo. There have only been a few reports of EBV genome mutations that promote the proliferation of EBV-infected cells. Our data imply that mutations of the viral genome can also contribute to EBV-associated tumor formation and proliferation.

Although deletions in the $\mathrm{Cp}$ region were associated with impaired transformation ability in previous studies [16], our dCp virus repeatedly demonstrated enhanced transformation efficiency. This may be due to differences in the deleted regions. The Cp deletion we used is very short and spans the transcription start site, making it likely that the region outside of the deletion contains a cis-acting sequence motif, which is crucial for transformation. The exact function of these regions needs to be clarified in further studies.

Transformation is generally mediated by enhancement of cellular growth and repression of cellular apoptosis. We focused on the contribution of the oncoprotein LMP2A and anti-apoptotic protein BHRF1 to B cell transformation, but neither could account for the enhanced transformation efficiency seen in the $\mathrm{dCp}$ virus. LMP2A gene was expressed abundantly in the dCp LCLs compared to WT (Figure 5). We still do not know why and how the LMP2 gene was overexpressed by deletion of the Cp, but the three-dimensional structure of the viral genome may play a role [26].

Disruption of BHRF1 had a very severe effect on B cell growth transformation compared to the mild effect seen in a previous report [24]. We speculate that this difference was attributable to the cells involved; the previous study used B cells derived from adenoids after T cell depletion, while we used PBMCs. The presence of germinal center B cells in adenoid tissue, and the depletion of $\mathrm{T}$ cells, may affect the severity of the response. 
Cells infected with the dBHRF1 virus showed severely impaired transformation efficiency compared to the WT virus, and the dCpdBHRF1 virus showed similar transformation efficiency to the $\mathrm{dCp}$ virus alone. This implies that, although BHRF1 does not play a role in the dCp-related higher transformation efficiency, the anti-apoptotic effect of BHRF1 could be compensated by $\mathrm{Cp}$ deletion. In this context, LMP2A was reported to induce degradation of anti-apoptotic proteins such as BHRF1, where BHRF1 targets protein Bim by activating ERK [27]. Elevated expression of LMP2A in the dCp virus-infected LCLs may affect this pathway. Additionally, the regulation of host genes by $\mathrm{Cp}$ deletion needs to be clarified in future studies.

In summary, we demonstrated that deletion of the Cp increased B cell growth transformation. This deletion was derived from a clinical sample and had the potential as a marker of malignancy. The role of $\mathrm{Cp}$ deletion in transformation efficiency suggests a novel tumorigenic mechanism, but further studies will be required to confirm this.

\section{Materials and Methods}

\subsection{Cell Culture and Reagents}

The cell lines HEK293, HEK293 EBV-BAC, and HEK293T were cultured in Dulbecco's modified Eagle's medium (Sigma-Aldrich, St. Louis, MO, USA) supplemented with $12 \%$ fetal bovine serum (FBS). Akata(-) cells were cultured in RPMI 1640 medium (SigmaAldrich) supplemented with $12 \%$ FBS. PBMCs were collected from healthy adult male donors who provided written informed consent, according to protocols approved by the Institutional Review Board of Nagoya University or Lonza Group AG. PBMCs and LCLs were maintained in RPMI 1640 medium (Sigma-Aldrich) supplemented with 12\% FBS with $1 \%$ nonessential amino acids (Sigma-Aldrich).

\subsection{Construction of the $d C p, d L M P 2 A$ and $d B H R F 1 E B V-B A C$ Genome and Transfection into HEK293 Cells}

The B95-8 EBV-BAC DNA sequence was a gift from W. Hammerschmidt [28]. To modify the EBV-BAC genome, homologous recombination was induced in E.coli, as described previously [29]. DNAs cassette containing neomycin-resistant and streptomycinsensitive genes $(\mathrm{Neo} / \mathrm{St})$, with homology arms adjacent to the target sequences of $\mathrm{Cp}$, LMP2A and BHRF1 were prepared by performing PCR using the rpsL-neo vector (Gene Bridges, Heidelberg, Germany) as template and primers, as follows: (Cp: forward 5'- CCTACGGGCGGGATTAATTACGCCTTGCTTACGCAAGCTCAGTTAATTCGCCCACGA CTTGGCCTGGT GATGATGGCGGGATC-3' ${ }^{\prime}$, reverse $5^{\prime}$-GCTGTTTCTTCAGTCCTAGAG GGAAGGAGAA TCACA TAAATTATTAATCTGCAAATAAAGTC AGAAGAA CTCGT CAAGAA GG-3', LMP2A: forward 5'-TGATCCTGTAGCGCCGCGGTTTCAGCATCACAG GTTATTTTGCCTGAAGCTTGCT GGGGCGTAGGCCTGGTGATGATGGCGGGATC-3', reverse $5^{\prime}$-GCCCTTATTATTGATGTGACTTG TGATGCAATAAATAAAAGTACAGATAGA TGGCACTCTTACTCAGAAGAACTCGTCAAGAAGG-3', BHRF1: forward 5'-CTGTAGTT CTGCGTTATCATGTGTTGCTTGAGGAGATAATTGAACGA AATTCAGAGACATGGCCT GGTGATGATGGCGGGATC-3' ${ }^{\prime}$, reverse $5^{\prime}$-AGTTAAAATCCAGATCC ACATGTTCGGTGT GTGTTATAAATCTGTTCCAAGTTTCTGTAACAGAAGAACTCGTCAAGAAGG-3').

TheseDNA cassettes were introduced into E. coli containing EBV-BAC by electroporation. Homologous recombination was induced by Red/ET, and E. coli harboring rearranged mutant EBV-BAC was selected by kanamycin. Homologous recombination was performed again, replacing the Neo/St containing DNA cassette with the target sequence.

The target DNA for Cp deletion was made by overlapping PCR, using the following primers: (overlapping template oligos: 5'-CCTACGGGCGGGATTAATTACGCCTTGCTTA CGCAAGCTCAGT TAATTCGCCCACGACTTCTTTATTTGC-3' , and 5'-GCTGTTTCTTCA GTCCTAGAGGGAAGGAG AATCACATAAATTATTAATCTGCAAATAAAGAAGTCGTG GG-3' , amplification primers 5'-CCT ACGGGCGGGATTAATTA-3', reverse 5'-GCTGTTTCT TCAGTCCTAGA- $3^{\prime}$ ). The target DNA for LMP2A deletion was also prepared by overlapping PCR, using the following primers (overlapping template oligos: 5'-TCCTGTAGCGCCG 
CGGTTTCAGCATCACAGGTTATTTTGCCTGAAGCTTGC TGGGGCGTAGTAAGAGTG C- $3^{\prime}$, and $5^{\prime}$-GCCCTTATTATTGATGTGACTTGTGATGCAATAAAT AAAAGTACAGATA GATGGCACTCTTACTACGCCCCAG- ${ }^{\prime}$, amplification primers $5^{\prime}$ - TGATC CTGTAGCGCC GCGG-3' ${ }^{\prime}$, reverse $5^{\prime}$ - GCCCTTATTATTGATGTGACT-3'). The targeting vector for the BHRF1 sequence with a stop codon was made by inverse PCR using the following primers: follows (forward 5'-CTGTAGTTCTGCGTTATCATGTGTTGCTTGAGGAGATAATTGAAC G AAATTCAGAGACATAATTACAGAAA-3' ${ }^{\prime}$, reverse 5'-AGTTAAAATCCAGATCCACAT GTTCGGTGTGTGTTATAAATC TGTTCCAAGTTTCTGTAATTATGTCTCTG-3').

E. coli containing dCp, dLMP2A and dBHRF1 EBV-BAC was selected by streptomycin. The rev strain for the $\mathrm{dCp}$ virus was constructed by reinserting the Neo/St cassette and then replacing it with the WT Cp sequence. The recombination integrity was checked by PCR, sequence analysis, and analysis of band patterns in electrophoresis of the BamHI- or EcoRI-digested viral genome.

HEK293 cells were transfected with the recombinant EBV-BAC DNA using FuGENE ${ }^{\circledR}$ HD transfection reagent (Promega, Madison, WI, USA). GFP-positive and hygromycin $(150 \mu \mathrm{g} / \mathrm{mL})$-resistant colonies were picked up for further analyses.

\subsection{Lytic Induction and Progeny Virus Titration}

The BZLF1 expression plasmid was prepared as reported previously [30,31]. In brief, HEK293 EBV-BAC cells were transfected with BZLF1-expressing plasmid using FuGENE ${ }^{\circledR}$ HD transfection reagent (Promega) to induce lytic cycle and progeny virus production. The supernatant containing progeny virus was concentrated by supercentrifuge and used to infect Akata(-) cells. After formalin fixation, the GFP-positive cell proportion was measured by FACS (Gallios, Beckman Coulter, Brea, CA, USA) for viral titration.

\subsection{B Cell Growth Transformation Assay}

Serial 10-fold dilutions of titer normalized virus solutions were used to infect $1.0 \times 10^{5}$ PBMCs in a 96-well plate with cyclosporine A. After 3 weeks, the $50 \%$ transforming dose was determined by counting the number of wells showing LCL growth. PBMCs were obtained from LONZA and PRECISION FOR MEDICINE.

\subsection{The EBV-Associated Lymphoproliferative Disorder Mouse Xenograft Model}

Human cord blood mononuclear cells $\left(1.0 \times 10^{7}\right)$ were infected with WT or dCp virus and immediately intraperitoneally injected into NOG mice $(n=6)$ [10]. On days 7, 14, 21 and 28, the body weight was measured and observed until these mice died because of EBV-associated lymphoproliferative disorder development. H-E staining was performed as described previously [10].

\subsection{RNA Sequencing (RNAseq) and Quantitative Reverse Transcription-Polymerase Chain Reaction ( $q R T-P C R)$}

Total RNA was isolated from LCLs using RNeasy mini kit (Qiagen). For RNAseq, the quality of the RNA was checked by the tape station system (Agilent Technologies), followed by poly(A) RNA enrichment using poly(A) RNA magnetic isolation module (NEB). The sequencing library was prepared using NEBNext Ultra II RNA prep kit for Illumina (NEB) and sequenced using HiSeq $\mathrm{X}$ next-generation sequencer (Illumina). Data were processed and expressed as fragments per kilobase of exon per million reads mapped (FPKM) as described [10]. The qRT-PCR reactions were carried out using a one-step SYBR PrimeScript RT-PCR Kit II (TaKaRa) and real-time PCR system 7300 (Thermo Fisher Scientific), as described [32].

\section{Conclusions}

Deletion of the Cp, originally found in EBV-positive DLBCL, appeared to increase growth transformation efficiency. Although the molecular mechanism of this phenomenon is still elusive, loss of part of the EBV gene may increase tumorigenicity. 
Author Contributions: Conceptualization, Y.O., T.M. and H.K.; methodology, S.M., F.H., T.W., Y.Y., Y.O., H.M.A.A.M. and Y.S.; validation, S.M., F.H., T.W., Y.Y., Y.O., H.M.A.A.M. and Y.S.; formal analysis, S.M., F.H., T.W., Y.Y., Y.O., H.M.A.A.M. and Y.S.; investigation, S.M., F.H., T.W., Y.Y., Y.O., H.M.A.A.M. and Y.S.; resources, T.M. and H.K.; data curation, T.M. and H.K.; writing-original draft preparation, S.M. and T.M.; writing—review and editing, S.M., Y.S., T.M. and H.K.; visualization, S.M., F.H. and T.M.; supervision, T.M. and H.K.; project administration, T.M. and H.K.; funding acquisition, T.M. and H.K. All authors have read and agreed to the published version of the manuscript.

Funding: This work was supported by grants-in-aid for Scientific Research from the Ministry of Education, Culture, Sports, Science and Technology (19K07580 to T.M., 19K22560 and 20H03493 to H.K.), Japan Agency for Medical Research and Development (AMED) (JP20wm0325012 to T.M.), the Takeda Science Foundation (to T.M.), and the Hori Sciences and Arts Foundation (to T.M., Y.S. and H.K.), and the General Assembly of the Japanese Association of Medical Sciences (to T.M.).

Institutional Review Board Statement: Ethical review and approval were not needed for this study, because this was a basic research that did not involve clinical specimens. PBMCs were commercially purchased. Animal experiments were carried out in accordance with the Guidelines for Animal Experimentation of Nagoya University, Japan.

Informed Consent Statement: Not applicable.

Data Availability Statement: Data are available upon request.

Acknowledgments: We thank Hammerschmidt, W.; Delecluse, H.J.; Kanda, T. and Tsurumi, T. for materials and discussions.

Conflicts of Interest: The authors declare no conflict of interest.

\section{References}

1. Murata, T.; Sato, Y.; Kimura, H. Modes of infection and oncogenesis by the Epstein-Barr virus. Rev. Med. Virol. 2014, 24, 242-253. [CrossRef] [PubMed]

2. Young, L.S.; Rickinson, A.B. Epstein-Barr virus: 40 years on. Nat. Rev. Cancer 2004, 4, 757-768. [CrossRef] [PubMed]

3. Cen, O.; Longnecker, R. Latent Membrane Protein 2 (LMP2). Curr. Top. Microbiol. Immunol. 2015, 391, 151-180. [CrossRef]

4. Wang, L.W.; Jiang, S.; Gewurz, B.E. Epstein-Barr Virus LMP1-Mediated Oncogenicity. J. Virol. 2017, 91, e01718-16. [CrossRef] [PubMed]

5. Shumilov, A.; Tsai, M.H.; Schlosser, Y.T.; Kratz, A.S.; Bernhardt, K.; Fink, S.; Mizani, T.; Lin, X.; Jauch, A.; Mautner, J.; et al. Epstein-Barr virus particles induce centrosome amplification and chromosomal instability. Nat. Commun. 2017, 8, 14257. [CrossRef]

6. Zhou, H.; Schmidt, S.C.; Jiang, S.; Willox, B.; Bernhardt, K.; Liang, J.; Johannsen, E.C.; Kharchenko, P.; Gewurz, B.E.; Kieff, E.; et al. Epstein-Barr virus oncoprotein super-enhancers control B cell growth. Cell Host Microbe 2015, 17, 205-216. [CrossRef]

7. Anastasiadou, E.; Stroopinsky, D.; Alimperti, S.; Jiao, A.L.; Pyzer, A.R.; Cippitelli, C.; Pepe, G.; Severa, M.; Rosenblatt, J.; Etna, M.P.; et al. Epstein-Barr virus-encoded EBNA2 alters immune checkpoint PD-L1 expression by downregulating miR-34a in B-cell lymphomas. Leukemia 2019, 33, 132-147. [CrossRef]

8. Bi, X.W.; Wang, H.; Zhang, W.W.; Wang, J.H.; Liu, W.J.; Xia, Z.J.; Huang, H.Q.; Jiang, W.Q.; Zhang, Y.J.; Wang, L. PD-L1 is upregulated by EBV-driven LMP1 through NF- $\mathrm{kB}$ pathway and correlates with poor prognosis in natural killer/T-cell lymphoma. J. Hematol. Oncol. 2016, 9, 109. [CrossRef]

9. Kelly, G.L.; Long, H.M.; Stylianou, J.; Thomas, W.A.; Leese, A.; Bell, A.I.; Bornkamm, G.W.; Mautner, J.; Rickinson, A.B.; Rowe, M. An Epstein-Barr virus anti-apoptotic protein constitutively expressed in transformed cells and implicated in burkitt lymphomagenesis: The Wp/BHRF1 link. PLoS Pathog. 2009, 5, e1000341. [CrossRef]

10. Okuno, Y.; Murata, T.; Sato, Y.; Muramatsu, H.; Ito, Y.; Watanabe, T.; Okuno, T.; Murakami, N.; Yoshida, K.; Sawada, A.; et al. Defective Epstein-Barr virus in chronic active infection and haematological malignancy. Nat. Microbiol. $2019,4,404-413$. [CrossRef]

11. Peng, R.J.; Han, B.W.; Cai, Q.Q.; Zuo, X.Y.; Xia, T.; Chen, J.R.; Feng, L.N.; Lim, J.Q.; Chen, S.W.; Zeng, M.S.; et al. Genomic and transcriptomic landscapes of Epstein-Barr virus in extranodal natural killer T-cell lymphoma. Leukemia 2019, 33, 1451-1462. [CrossRef] [PubMed]

12. Woisetschlaeger, M.; Jin, X.W.; Yandava, C.N.; Furmanski, L.A.; Strominger, J.L.; Speck, S.H. Role for the Epstein-Barr virus nuclear antigen 2 in viral promoter switching during initial stages of infection. Proc. Natl. Acad. Sci. USA 1991, 88, 3942-3946. [CrossRef]

13. Woisetschlaeger, M.; Yandava, C.N.; Furmanski, L.A.; Strominger, J.L.; Speck, S.H. Promoter switching in Epstein-Barr virus during the initial stages of infection of B lymphocytes. Proc. Natl. Acad. Sci. USA 1990, 87, 1725-1729. [CrossRef] 
14. Lin, X.; Tsai, M.H.; Shumilov, A.; Poirey, R.; Bannert, H.; Middeldorp, J.M.; Feederle, R.; Delecluse, H.J. The Epstein-Barr Virus BART miRNA Cluster of the M81 Strain Modulates Multiple Functions in Primary B Cells. PLoS Pathog. 2015, 11, e1005344. [CrossRef] [PubMed]

15. Swaminathan, S. Characterization of Epstein-Barr virus recombinants with deletions of the BamHI C promoter. Virology 1996, 217, 532-541. [CrossRef] [PubMed]

16. Tierney, R.J.; Nagra, J.; Rowe, M.; Bell, A.I.; Rickinson, A.B. The Epstein-Barr virus BamHI C promoter is not essential for B cell immortalization in vitro, but it greatly enhances B cell growth transformation. J. Virol. 2015, 89, 2483-2493. [CrossRef] [PubMed]

17. Ba Abdullah, M.M.; Palermo, R.D.; Palser, A.L.; Grayson, N.E.; Kellam, P.; Correia, S.; Szymula, A.; White, R.E. Heterogeneity of the Epstein-Barr Virus (EBV) Major Internal Repeat Reveals Evolutionary Mechanisms of EBV and a Functional Defect in the Prototype EBV Strain B95-8. J. Virol. 2017, 91. [CrossRef]

18. Longnecker, R.; Miller, C.L.; Tomkinson, B.; Miao, X.Q.; Kieff, E. Deletion of DNA encoding the first five transmembrane domains of Epstein-Barr virus latent membrane proteins 2A and 2B. J. Virol. 1993, 67, 5068-5074. [CrossRef]

19. Brielmeier, M.; Mautner, J.; Laux, G.; Hammerschmidt, W. The latent membrane protein 2 gene of Epstein-Barr virus is important for efficient B cell immortalization. J. Gen. Virol. 1996, 77 Pt 11, 2807-2818. [CrossRef]

20. Caldwell, R.G.; Wilson, J.B.; Anderson, S.J.; Longnecker, R. Epstein-Barr virus LMP2A drives B cell development and survival in the absence of normal B cell receptor signals. Immunity 1998, 9, 405-411. [CrossRef]

21. Flanagan, A.M.; Letai, A. BH3 domains define selective inhibitory interactions with BHRF-1 and KSHV BCL-2. Cell Death Differ 2008, 15, 580-588. [CrossRef]

22. Desbien, A.L.; Kappler, J.W.; Marrack, P. The Epstein-Barr virus Bcl-2 homolog, BHRF1, blocks apoptosis by binding to a limited amount of Bim. Proc. Natl. Acad. Sci. USA 2009, 106, 5663-5668. [CrossRef]

23. Kvansakul, M.; Wei, A.H.; Fletcher, J.I.; Willis, S.N.; Chen, L.; Roberts, A.W.; Huang, D.C.; Colman, P.M. Structural basis for apoptosis inhibition by Epstein-Barr virus BHRF1. PLoS Pathog. 2010, 6, e1001236. [CrossRef]

24. Altmann, M.; Hammerschmidt, W. Epstein-Barr virus provides a new paradigm: A requirement for the immediate inhibition of apoptosis. PLoS Biol. 2005, 3, e404. [CrossRef]

25. Watanabe, A.; Maruo, S.; Ito, T.; Ito, M.; Katsumura, K.R.; Takada, K. Epstein-Barr virus-encoded Bcl-2 homologue functions as a survival factor in Wp-restricted Burkitt lymphoma cell line P3HR-1. J. Virol. 2010, 84, 2893-2901. [CrossRef]

26. Tempera, I.; Lieberman, P.M. Epigenetic regulation of EBV persistence and oncogenesis. Semin. Cancer Biol. 2014, 26, 22-29. [CrossRef]

27. Iwakiri, D.; Minamitani, T.; Samanta, M. Epstein-Barr virus latent membrane protein 2A contributes to anoikis resistance through ERK activation. J. Virol. 2013, 87, 8227-8234. [CrossRef]

28. Delecluse, H.J.; Hilsendegen, T.; Pich, D.; Zeidler, R.; Hammerschmidt, W. Propagation and recovery of intact, infectious Epstein-Barr virus from prokaryotic to human cells. Proc. Natl. Acad. Sci. USA 1998, 95, 8245-8250. [CrossRef]

29. Murata, T.; Isomura, H.; Yamashita, Y.; Toyama, S.; Sato, Y.; Nakayama, S.; Kudoh, A.; Iwahori, S.; Kanda, T.; Tsurumi, T. Efficient production of infectious viruses requires enzymatic activity of Epstein-Barr virus protein kinase. Virology 2009, 389, 75-81. [CrossRef]

30. Konishi, N.; Narita, Y.; Hijioka, F.; Masud, H.M.A.A.; Sato, Y.; Kimura, H.; Murata, T. BGLF2 Increases Infectivity of Epstein-Barr Virus by Activating AP-1 upon. mSphere 2018, 3, e00138-18. [CrossRef]

31. Al Masud, H.M.A.; Watanabe, T.; Yoshida, M.; Sato, Y.; Goshima, F.; Kimura, H.; Murata, T. Epstein-Barr Virus BKRF4 Gene Product Is Required for Efficient Progeny Production. J. Virol. 2017, 91, e00975-17. [CrossRef]

32. Ichikawa, T.; Okuno, Y.; Sato, Y.; Goshima, F.; Yoshiyama, H.; Kanda, T.; Kimura, H.; Murata, T. Regulation of Epstein-Barr Virus Life Cycle and Cell Proliferation by Histone H3K27 Methyltransferase EZH2 in Akata Cells. mSphere 2018, 3, e00478-18. [CrossRef] 